\title{
Endoscopic ultrasound guided fine needle biopsy (EUS-FNB) from peritoneal lesions: a prospective cohort pilot study
}

Pradermchai Kongkam ${ }^{1,2}$, Theerapat Orprayoon ${ }^{1,2^{*}}$, Sirilak Yooprasert ${ }^{1}$, Nakarin Sirisub ${ }^{3}$, Naruemon Klaikaew ${ }^{4}$, Anapat Sanpawat ${ }^{4}$, Shahram Safa ${ }^{1,2}$, Wiriyaporn Ridtitid ${ }^{1}$, Pinit Kullavanijaya ${ }^{1}$ and Rungsun Rerknimitr ${ }^{1}$

\begin{abstract}
Background: Diagnostic laparoscopy is often a necessary, albeit invasive, procedure to help resolve undiagnosed peritoneal diseases. Previous retrospective studies reported that EUS-FNA is feasible on peritoneal and omental lesions, however, EUS-FNA provided a limited amount of tissue for immunohistochemistry stain (IHC).

Aim: This pilot study aims to prospectively determine the effectiveness of EUS-FNB regarding adequacy of tissue for IHC staining, diagnostic rate and the avoidance rate of diagnostic laparoscopy or percutaneous biopsy in patients with these lesions.

Methods: From March 2017 to June 2018, patients with peritoneal or omental lesions identified by CT or MRI at the King Chulalongkorn Memorial Hospital, Bangkok, Thailand were prospectively enrolled in the study. All Patients underwent EUS-FNB. For those with negative pathological results of EUS-FNB, percutaneous biopsy or diagnostic laparoscopy was planned. Analysis uses percentages only due to small sample sizes.

Results: A total of 30 EUS-FNB passes were completed, with a median of 3 passes (range 2-3 passes) per case. For EUS-FNB, the sensitivity, specificity, PPV, NPV and accuracy of EUS-FNB from peritoneal lesions were $63.6 \%, 100 \%$, $100 \%, 20 \%$ and $66.7 \%$ respectively. Adequate tissue for $\mathrm{IHC}$ stain was found in $25 / 30$ passes (80\%). The tissues from EUS results were found malignant in $7 / 12$ patients (58.3\%). IHC could be done in 10/12 patients (83.3\%). Among the five patients with negative EUS results, two underwent either liver biopsy of mass or abdominal paracentesis, showing gallbladder cancer and adenocarcinoma. Two patients refused laparoscopy due to advanced pancreatic cancer and worsening ovarian cancer. The fifth patient had post-surgical inflammation only with spontaneous resolution. The avoidance rate of laparoscopic diagnosis was 58.3\%. No major adverse event was observed.
\end{abstract}

Conclusions: EUS-FNB from peritoneal lesions provided sufficient core tissue for diagnosis and IHC. Diagnostic laparoscopy can often be avoided in patients with peritoneal lesions.

Keywords: Peritoneal carcinomatosis, Carcinomatosis peritonei, Omental cake, Omentum, Endoscopic ultrasound (EUS), Endoscopic ultrasound-guided fine needle aspiration (EUS-FNA), Endoscopic ultrasound-guided fine needle biopsy (EUS-FNB), Abdominal paracentesis, Ascites, Peritoneal space, Peritoneal ligament

\footnotetext{
*Correspondence: platy_pt1301@hotmail.com

1 Excellence Center for Gastrointestinal Endoscopy, King

Chulalongkorn Memorial Hospital, Thai Red Cross Society, and Division

of Gastroenterology, Department of Medicine, Faculty of Medicine,

Chulalongkorn University, Bangkok, Thailand

Full list of author information is available at the end of the article
}

(C) The Author(s) 2021. Open Access This article is licensed under a Creative Commons Attribution 4.0 International License, which permits use, sharing, adaptation, distribution and reproduction in any medium or format, as long as you give appropriate credit to the original author(s) and the source, provide a link to the Creative Commons licence, and indicate if changes were made. The images or other third party material in this article are included in the article's Creative Commons licence, unless indicated otherwise in a credit line to the material. If material is not included in the article's Creative Commons licence and your intended use is not permitted by statutory regulation or exceeds the permitted use, you will need to obtain permission directly from the copyright holder. To view a copy of this licence, visit http://creativecommons.org/licenses/by/4.0/. The Creative Commons Public Domain Dedication waiver (http://creativeco mmons.org/publicdomain/zero/1.0/) applies to the data made available in this article, unless otherwise stated in a credit line to the data. 


\section{Key summary}

1. Summarise the established knowledge on this subject

- Diagnostic laparoscopy is the gold standard test for diagnosing the cause of omental cake

- EUS-FNA can provide cyto-pathological diagnosis of omental cake in the majority of patients but is an unproven technique for core biopsy with immunohistochemical staining.

2. What are the significant and/or new findings of this study?

- EUS-FNB can provide sufficient tissue for pathological diagnosis with immunohistochemical staining in the majority of patients with omental cake.

\section{Introduction}

Peritoneal or omental lesions including peritoneal thickening, peritoneal mass or nodule, omental cake or mass can result from a variety of diseases. To unify terminologies, lesions are universally referred to as peritoneal lesions in our study. To diagnose etiologies of peritoneal lesions, several modalities including radiological findings, tissue biopsy under radiological guidance, and diagnostic laparoscopy have been used. Cross-sectional imaging findings of peritoneal lesions alone are too non-specific to provide an etiological diagnosis. Often, this method may underestimate peritoneal disease burden, since it has limitations in the detection of a small volume peritoneal implants or ascites [1]. Presumptive diagnosis of malignant peritoneal lesions in known primary cancer is not always correct as these lesions can arise from a second primary peritoneal malignancy in $10 \%$ of cases [2]. A definite diagnosis of peritoneal lesions is hence required for precise clinical management.

The limitations of current procedures underscore the need for biopsy confirmation of peritoneal lesions. The rapid advancements in cancer therapy along with greater focus on patient outcomes demand an ever-increasing need for precise staging and exact diagnosis. Immunohistochemical (IHC) staining and molecular analysis, which can be more easily examined with core tissue than cytology, has been increasingly requested by oncologists. Diagnostic laparoscopy is considered the gold standard in the diagnosis of peritoneal lesions with a sensitivity $86 \%$ and an ability to provide core tissue for IHC staining [3]. Nevertheless, it is an invasive surgical procedure. A simple and low-cost bedside procedure like abdominal paracentesis may also be helpful in the diagnosis of malignancy-related ascites. Unfortunately, the sensitivity of ascitic fluid cytology for detecting malignancy is much lower at $57-67.1 \%$ and paracentesis does not provide core tissue [4-8]. Paracentesis is also not feasible in cases without ascites, which accounts for two-thirds of patients with peritoneal carcinomatosis [9]. While CTguided percutaneous peritoneal lesions biopsy has also been reported with a sensitivity of $89.5 \%$, this procedure necessitates radiation exposure and is problematic for deeply located target lesions in the abdomen [10].

More recently, EUS-guided fine needle aspiration (EUS-FNA) of peritoneal lesions has been shown to be feasible, with a sensitivity between $90-100 \%$ in several case reports as well as in a large retrospective study of 98 patients by Levy et al. [11-13]. Nevertheless, it is not practical to use tissue from EUS-FNA for IHC staining due to the relatively small volume of tissue collected compared to a EUS-guided fine needle biopsy (EUS-FNB) [14]. To the best of our knowledge, no study of EUS-FNB for diagnosis of peritoneal lesions has been undertaken. This prospective study aimed to fill this research gap and evaluate the adequacy of tissue for immunohistochemical staining of EUS-FNB from peritoneal lesions and determine the diagnostic yield.

\section{Methods \\ Patients}

From March 2017 to June 2018, consecutive patients aged $>18$ years with peritoneal lesions identified by CT or MRI of the abdomen at King Chulalongkorn Memorial Hospital, Bangkok, Thailand were enrolled in the study. All patients signed a consent form before undergoing the procedure. CT or MRI revealed features of peritoneal lesions including peritoneal nodules, plaques or sheets of soft tissue in the sub-hepatic area, anterior abdominal wall, paracolic gutter and cul-de-sac, stranding around and thickening of the omentum (omental cake), and thickening, stranding and distortion of the mesentery with or without presence of ascites. The exclusion criteria included uncorrectable coagulopathy, esophageal obstruction, undetectable target lesions by EUS, poor ECOG score ( $>2)$ signifying being unfit for EUS procedure, intervening tumor in puncture site, and pregnancy.

Patient baseline characteristics including age, sex, comorbidities, previous diagnosis and treatment of malignancy, imaging features of abdomen, EUS findings of lesions, number of passes, procedural time, results of pathology and complications were recorded and analyzed.

This study used a prospective design to evaluate the adequacy of tissue for immunohistochemical staining of EUS-FNB and the diagnostic yield of EUS-FNB in patients with undiagnosed peritoneal lesions. The study was approved by the Institutional Review Board of Chulalongkorn University in Bangkok, Thailand with 
IRB number $422 / 60$. The clinical trial number is TCTR 20170424001. We confirmed that all methods were carried out in accordance with relevant guidelines and regulations in the ethical approval and consent to participate subsection of declaration.

\section{Endoscopic techniques}

Prophylactic antibiotics were administered before performing the procedure. An EUS examination of the pancreas and other intra-abdominal organs was performed using a linear echoendoscope (EG-3270UK: Pentax Corporation and Ultrasound Scanner PREIRUS: Hitachi). Once identified, EUS-FNB from the peritoneal lesions was performed with a 20-gauge needle (EchoTip ProCore 20 gage needle; Cook Medical, Limerick, Ireland). This was done in order to obtain sufficient tissue for pathology, not only for cytology. Specimens obtained from EUS-FNB were placed on a glass slide for gross visualization to evaluate adequacy of specimens as described by Iwashita et al. [15]. Adequacy was scored following a protocol published by Wang et al. [16] EUS-FNB was repeated up to four times until the specimen seemed adequate by gross visualization of white matter in the specimen as previously described. (Additional file 1: Video 1) If suspicious lymph nodes or primary lesions were identified, these lesions were also sampled after EUS-FNB of the peritoneal lesions. The results of the biopsy from these lymph nodes or lesions were used as part of the final diagnosis. To perform EUS-FNB from target peritoneal lesions, endosonographer avoided intervening tumors or other organs such as the colonic wall.

\section{Diagnosis}

Pathology results were interpreted later at the pathological laboratory room by a gastrointestinal pathologist. Specimens were evaluated for tissue adequacy and cytopathological results. Adequacy of specimens was graded by a score previously described [16]. We used the result of the surgical or EUS-FNB pathology as the reference standard. Only a definite pathological interpretation of malignancy was considered positive, while all other suspicious or atypical types were reported as negative. If the pathology result was positive, the final diagnosis was malignant peritoneal lesions. If the result was negative or suspicious, diagnostic laparoscopy or percutaneous biopsy was planned. This approach is similar to EUS nodal staging in patients with lung cancer where patients with negative EUS-FNA from mediastinal lymph node are consequently advised to undergo mediastinoscopy [17]. In patients deemed unfit for or denied further diagnostic tests, a minimum six-month follow-up for clinical symptoms, radiology and blood tests was mandatory before making a presumptive diagnosis. Diagnostic yield of EUS-FNB and adequacy of tissue for immunohistochemical staining of EUS-FNB from peritoneal lesions were calculated as the primary endpoint.

A definite diagnosis on the etiology of the peritoneal lesion was made by cytological or histological interpretation of EUS-FNB or surgical specimens (Figs. 1, 2, 3). As this was a prospective study, all patients were encouraged to have a pathological diagnosis. In cases where no further intervention was done, a probable diagnosis was determined by clinical criteria and monitoring for disease progression such as increased lesion size with or without clinical deterioration, evidenced by cross-sectional

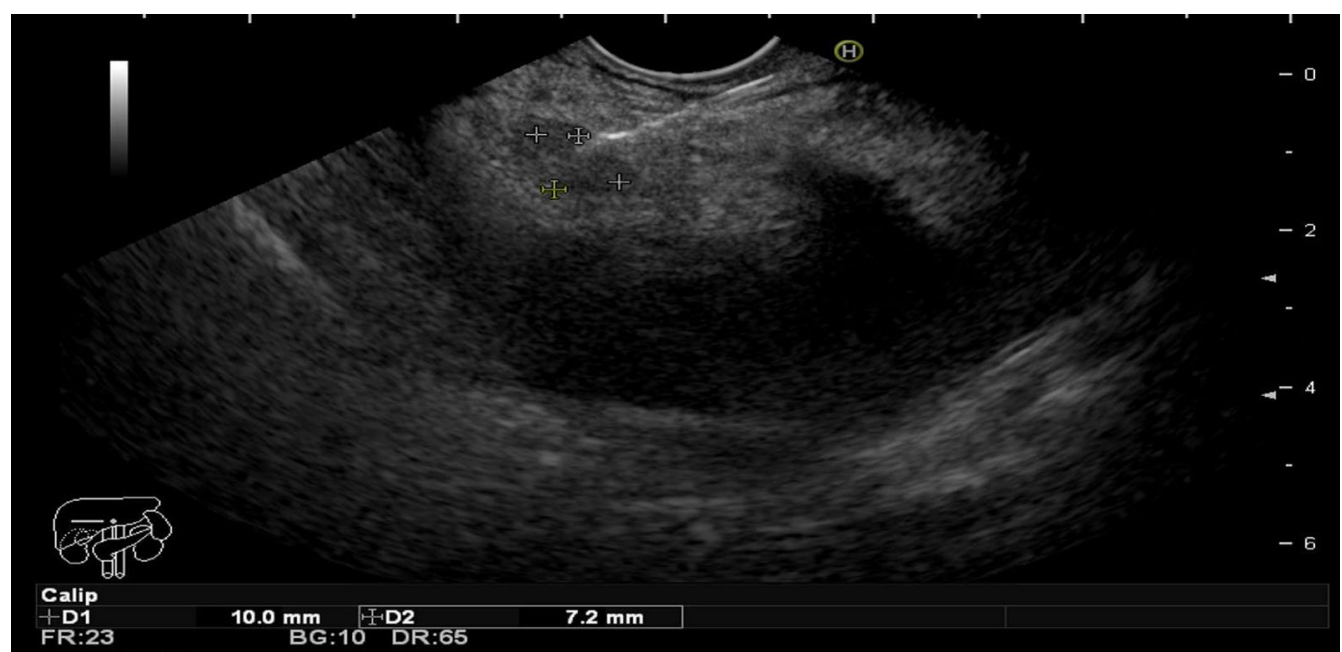

Fig. 1 Demonstrated a hypoechoic lesion in hyperechoic thickening peritoneum. The lesion was biopsy with a 20-gauge needle (EchoTip ProCore 20 gage needle; Cook Medical, Limerick, Ireland) 


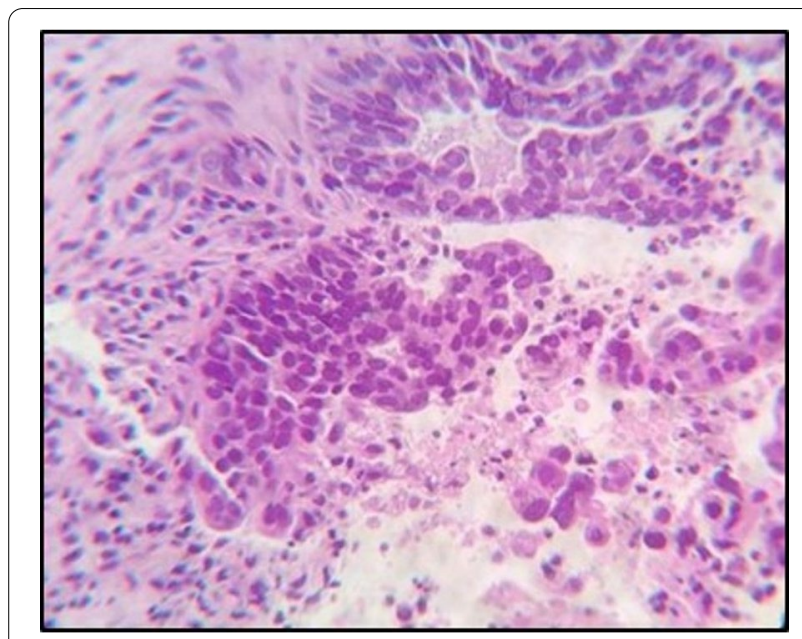

Fig. 2 Showed peritoneal tissue from a 20G endoscopic ultrasound guided fine needle biopsy needle. Histochemical staining showed metastatic tumor sheet

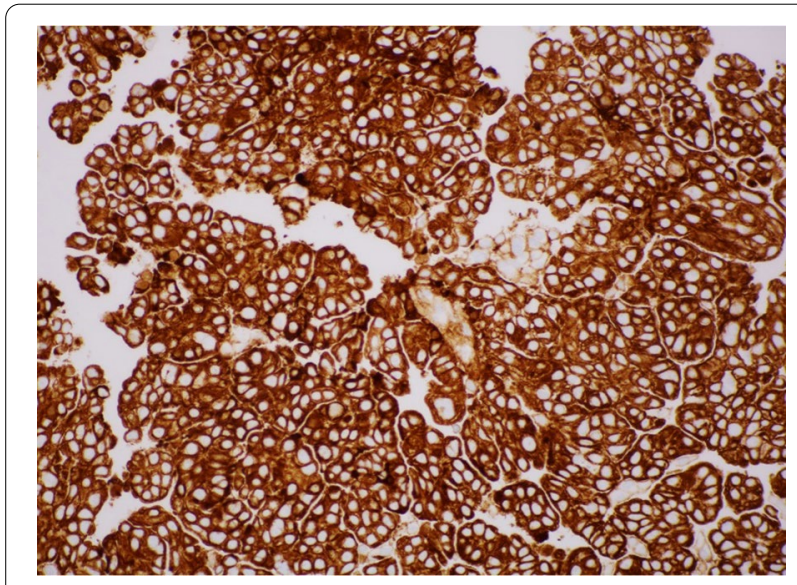

Fig. 3 Showed positive results of immunohistochemical staining from tissue obtained from a $20 \mathrm{G}$ endoscopic ultrasound guided fine needle biopsy needle

imaging, or treatment response over a 6-month followup. This criterion of probable diagnosis is like previous EUS studies [18].

\section{Follow up}

All patients were closely followed-up after the procedure for possible complications. Patients with positive EUS-FNB results were treated as having peritoneal malignancy. Results were sent to a multidisciplinary team. Impact on clinical management, particularly decision making for diagnostic laparoscopy from EUS-FNB results of peritoneal lesions, was recorded. Patients with negative EUS-FNB results proceeded to diagnostic laparoscopy. Those who did not consent to laparoscopy were advised to undergo percutaneous biopsy as a second option. Patients who refused both options were followedup clinically.

\section{Statistical analysis}

Continuous variables were expressed as mean \pm SD. Categorical variables were reported as frequency (\%). For the diagnostic study, sensitivity, specificity, accuracy, positive predictive value (PPV) and negative predictive value (NPV) were calculated. Chi-square and T-test were used when appropriate. SPSS version 22.0 was used for analysis.

\section{Results}

\section{Patient characteristics}

From March 2017 to June 2018, 23 patients met inclusion criteria. Eleven of them were excluded with reasons including poor performance status $(n=4)$, undetectable lesions during EUS $(n=4)$, intervening tumor in puncture site $(n=1)$, uncontrolled infection $(n=1)$, and esophageal obstruction $(\mathrm{n}=1)$. Eventually, 12 patients with CT findings suspicious for peritoneal lesions were enrolled in the study. Patients had a mean age of $62.9 \pm 9$ years with an equal number of males and females. The presenting symptoms included abdominal pain $(n=6 ; 50 \%)$, weight loss $(n=4 ; 33.3 \%)$ and abdominal distension $(n=2 ; 16.7 \%)$. The CT findings found in the patients were soft tissue nodules/mass deposit in peritoneum $(\mathrm{n}=11 ; 91.7 \%)$, ascites $(\mathrm{n}=11 ; 91.7 \%)$, omental cake $(n=4 ; 33.3 \%)$, and mesenteric stranding $(n=2$; $16.7 \%)$. Number of abnormal CT findings in 12 patients were: 1 abnormal CT finding $(\mathrm{n}=2), 2 \mathrm{CT}$ findings $(n=6) ; 3$ CT findings $(n=2)$; and 4 CT findings $(n=2)$. Patient's demographic data and clinical presentation are shown in Table 1 . Ascites was demonstrated by CT in 11 from 12 patients (91.7\%). Abdominal paracentesis was feasible in 9 patients (75\%). Paracentesis was not performed in 3 patients because of insufficient amount of ascites (Table 2).

\section{Diagnosis}

Malignant peritoneal lesions were found in 11 patients with one patient having benign lesions. Procedures used in malignancy diagnoses included cytology from pathology by EUS-FNB $(n=7)$, repeat abdominal paracentesis $(\mathrm{n}=1)$, percutaneous liver biopsy $(\mathrm{n}=1)$, and adequate follow up with evidence of disease progression over a 6 -month follow-up $(n=2)$. In a benign peritoneal lesion, etiology was identified post-surgery $(n=1)$. In the benign peritoneal lesion, the patient received follow up for more than 1 year (Fig. 4). 
Table 1 Demographic data and clinical presentation of study patients

\begin{tabular}{|c|c|c|}
\hline & & $\begin{array}{l}\text { EUS-FNB } \\
N=12 \\
(\%)^{*}\end{array}$ \\
\hline \multirow[t]{2}{*}{ Gender } & Male & $6(50)$ \\
\hline & Female & $6(50)$ \\
\hline Age, years (mean $+S D)$ & & $62.9 \pm 9$ \\
\hline \multirow[t]{4}{*}{ Presenting symptoms } & Weight loss & $4(33.3)$ \\
\hline & Abdominal Pain & $6(50)$ \\
\hline & Abdominal Distention & $2(16.7)$ \\
\hline & Jaundice & $0(0)$ \\
\hline \multirow[t]{4}{*}{ CT findings ${ }^{\#}$} & Soft tissue nodules/mass deposit in peritoneum & $11(91.7)$ \\
\hline & Ascites & $11(91.7)$ \\
\hline & Omental cake & $4(33.3)$ \\
\hline & Mesenteric stranding & $2(16.7)$ \\
\hline \multirow[t]{2}{*}{ EUS findings } & Thickened hyperechoic omental cake & $1(8.3)$ \\
\hline & Hypoechoic nodules/deposit in peritoneum or omentum & $11(91.7)$ \\
\hline
\end{tabular}

${ }^{*}$ All values reported as $\mathrm{n}(\%)$, except for age

\# Small ascites defined by shortest thickness of ascites from abdominal wall $<3 \mathrm{~mm}$ in CT or MRI

Table 2 Detection of ascites and performance of abdominal paracentesis

\begin{tabular}{lll}
\hline & & EUS-FNB (N=12 (\%)) \\
\hline Ascites & Detected on CT & $11(91.7)$ \\
& Detected on EUS & $12(100.0)$ \\
Abdominal Paracen- & Feasible & $9(75.0)$ \\
tesis & Not feasible & $3(25.0)$ \\
\hline
\end{tabular}

One from 9 patients who underwent abdominal paracentesis had a positive malignant cell from ascitic fluid

\section{EUS-FNB}

All FNB needle punctures were inserted through the stomach. There were a total of 30 passages with each patient receiving two or three passes. The tissues from EUS results found malignancy in 7 patients. The laparoscopic avoidance rate is $7 / 12(58.3 \%)$. IHC stain was achieved in $10 / 12$ patients $(83.3 \%)$. The sensitivity, specificity, PPV, NPV and accuracy of EUS-FNB from peritoneal lesions were $63.6 \%, 100 \%, 100 \%, 20 \%$ and $66.7 \%$, respectively (Fig. 1). The positive EUS-FNB finding for malignant lesions occurred in 17 passages with nearly all from hypoechoic lesions in 16/17 lesions (94.1\%) and one from hyperechoic lesions.

Definite pathological diagnosis of malignant peritoneal lesion from the EUS-FNB group was made in 9/12 patients. Seven patients were diagnosed from EUS-FNB specimens. One patient was diagnosed with adenocarcinoma with repeated abdominal paracentesis. Another patient was sent for liver biopsy and diagnosed with gallbladder carcinoma. The other three patients were provided clinical follow-up. One patient died after 1 month of follow-up. One patient experienced clinical worsening with an increase of ascites. Another patient clinically resolved and was diagnosed with post-operative inflammation (Table 3).

One EUS-FNB patient had an incident of abdominal pain which responded to analgesic drug regimen. Another patient experienced obscure anemia needing two units of red cell transfusion which was classified as moderate severity according to a lexicon for endoscopic adverse events [19].

\section{Discussion}

EUS-FNA and EUS-FNB have been world widely used for tissue diagnosis of solid pancreatic lesions over the last 2 decades. Varying designs and diameters of EUS-FNA and EUS-FNB needles have been developed and tested to be used properly in different kinds of lesions and circumstances. For example, a meta-analysis of EUS-FNA from solid pancreatic lesions showed no superiority of 25 - over 22-gauge needles [20]. In addition to solid pancreatic lesions, several studies have been done and demonstrated that EUS-FNB can provide high diagnostic yield in various targets e.g., subepithelial lesions, liver parenchyma, etc. [21, 22]. Nowadays, EUS technique has been expanded to more therapeutic options [23].

The design of this current study is to evaluate the clinical use of EUS-FNB for peritoneal lesions in similar fashion to the use of EUS for mediastinal staging of lung cancer where diagnostic mediastinoscopy is performed only in cases of negative EUS-FNB. Results from this 


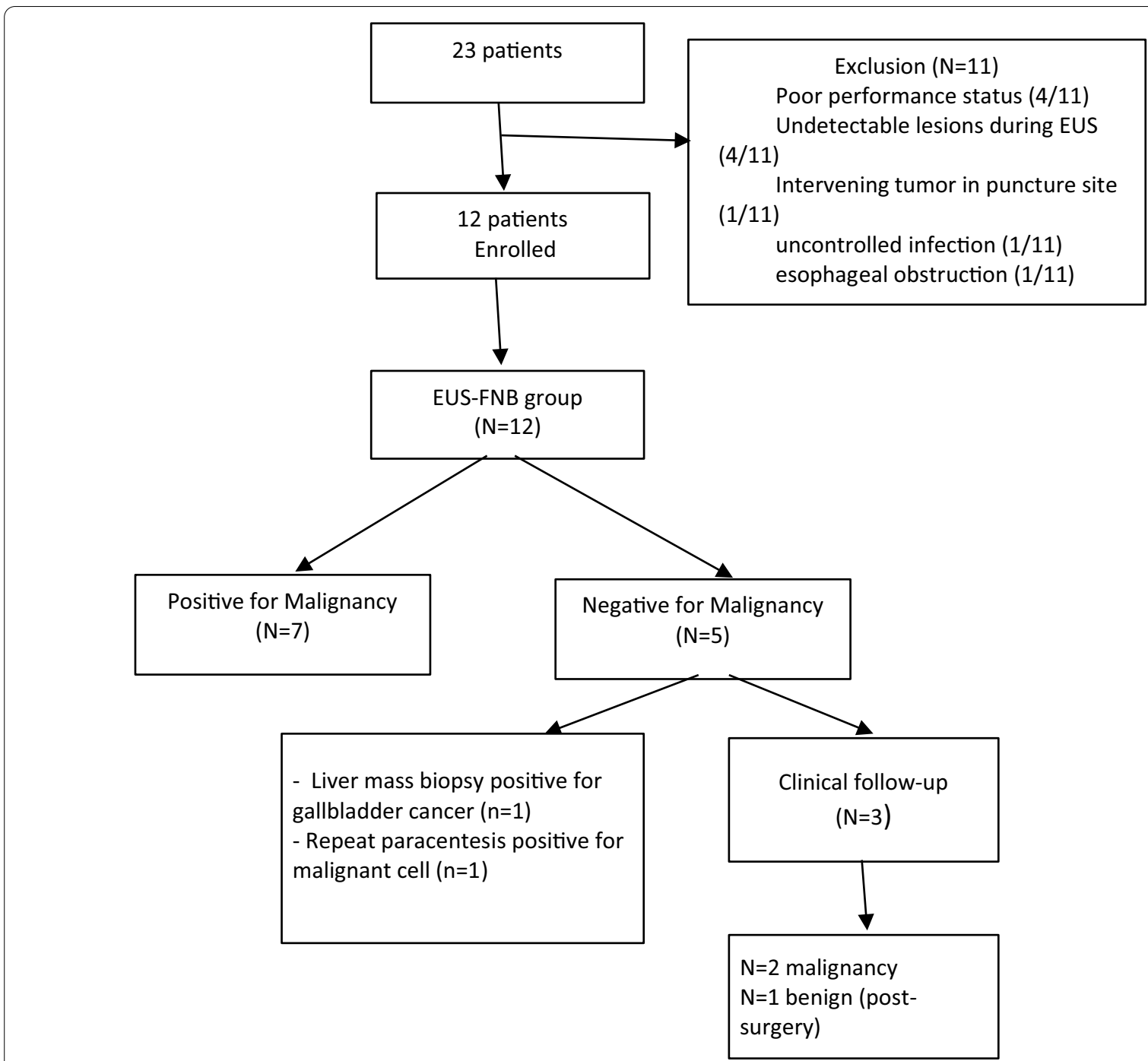

Fig. 4 This flow chart summarized overall results of all enrolled patients

Table 3 Final diagnosis in all patients

\begin{tabular}{ll}
\hline & EUS-FNB \\
& $\mathbf{N}=\mathbf{1 2}(\%)^{*}$ \\
\hline Pancreatic cancer & $4(33.3)$ \\
Gallbladder cancer & $1(8.3)$ \\
Gynecologic malignancy & $3(25)$ \\
Cholangiocarcinoma & $1(8.3)$ \\
Hepatocellular carcinoma & $1(8.3)$ \\
Carcinoma unknown primary & $1(8.3)$ \\
Benign disease & $1(8.3)$ \\
\hline
\end{tabular}

*All values reported as $\mathrm{n}(\%)$

present study show an impressive avoidance rate of diagnostic laparoscopy or percutaneous biopsy of 58.3\%. Clinicians who are treating patients with peritoneal lesions should consider EUS-FNB as a first-line test before diagnostic laparoscopy. This guidance is supported by results of previous studies that used EUS-FNA for diagnosing etiologies of peritoneal lesions including Levy et al., which demonstrated that EUS-FNA can detect peritoneal carcinomatosis better than CT/MRI with high sensitivity, specificity, and accuracy rate at $91 \%, 100 \%$, and $94 \%$, respectively, as compared to $28 \%, 85 \%$, and $47 \%$, respectively, for CT/MRI. In a retrospective study of 12 patients, Rana et al. found that EUS-FNA from peritoneal lesions could be performed safely and was able to diagnose malignant and benign peritoneal nodules in $83 \%$ $(10 / 12)$ of patients [24].

Most patients with peritoneal lesions either due to benign or malignant causes produce ascites detectable by $\mathrm{CT}$, which leads to abdominal paracentesis as a conventional diagnostic test. However, in two large retrospective 
studies, smaller amounts of ascites were undetected by CT including 6.5\% (52/798) and 21.8\% (12/55) [6, 11]. This current study confirmed this finding. This suggests that for patients with small amounts of ascites, even with a negative CT, EUS should be performed to confirm absence or presence of ascites because ascites can be an important diagnostic clue, and in malignant conditions can upstage the disease. Moreover, diagnosis of radiographic occult ascites in patients suspected for peritoneal carcinomatosis resulted in more accurate cancer staging and determination of resectability status [25]. Malignancy-related ascites account for about $7 \%$ of cases of ascites, with two-thirds of these patients having peritoneal carcinomatosis, which underscores the need for an accurate diagnosis to guide clinical management [26, 27].

In patients with peritoneal lesions and ascites with a negative result of abdominal paracentesis, diagnostic laparoscopy is often the next step for making a diagnosis of etiology. Unfortunately, diagnostic laparoscopy is typically an inpatient procedure requiring admission with a complication rate of $2-3 \%$ [28]. Results from this current study showed that EUS-FNB from peritoneal lesions is feasible and effective for diagnostic purposes without any serious complication. In addition, it is an outpatient procedure requiring no intensive post-procedural care and general anesthesia. Clinical application from results of this study suggests that EUS-FNB for peritoneal lesions might be considered as an initial diagnostic test before laparoscopy.

Regarding the endoscopic ultrasound findings of peritoneal lesions, Levy et al. stated that "most malignantappearing peritoneal anomalies were solid, hypoechoic masses, thickening or nodularity in the peritoneum and/ or omentum." This observation was also noted in our study where most cases with positive results were taken from hypoechoic peritoneal lesions. Based on the findings from these two studies, we suggest endosonographers performing the EUS-FNB of peritoneal lesions to select hypoechoic lesions as the target. However, with a limitation of reviewing all EUS imaging as a retrospective review, we did not have enough information to compare the sensitivity of EUS-FNB for hypoechoic versus hyperechoic lesions. Future systematic study is needed to clarify this point.

Although EUS-FNB has demonstrated a high diagnostic accuracy rate even without rapid on-site evaluation from solid pancreatic lesions. In this current study, the sensitivity of EUS-FNB for peritoneal lesions was only $63.6 \%$ which was relatively low. This might be explained by the different design and diameter of EUSFNB needles. Some previous studies showed a higher tissue acquisition rate of EUS-FNB from solid pancreatic lesions in new generation end -cutting needles (e.g., Fork-tip or Franseen needle) than side-fenestrated ones
$[29,30]$. Another new technique of tissue sampling has been introduced as through-the-needle microforceps biopsy (TTNB). A recent meta-analysis showed the sample adequacy of TTNB in pancreatic cyst was $85.3 \%$. The pooled diagnostic accuracy rate, sensitivity, and specificity of TTNB were $78.8 \%, 82.2 \%$, and $96.8 \%$ respectively [31]. Moreover, a study of EUS-TTNB revealed high interobserver agreement among pathologists [32]. Another recent case series demonstrated an impressive diagnostic performance for carcinomatosis peritonei [33]. In the future, it is interesting to compare diagnostic rate among different types of EUS-FNB needles with a fashion of head-to-head comparison study.

This study shows only one adverse event occurred with one patient experiencing obscure anemia needing 2 units of red cell transfusion; which was classified as moderate severity [19]. In comparison with the diagnostic laparoscopy, EUS-FNB is less invasive and an out-patient procedure but it is still too early to compare adverse event rate because the number of cases in this current study is too small. Nevertheless, EUS-FNB usage for peritoneal lesions has become an interesting alternative diagnostic tool compared with diagnostic laparoscopy.

The limitations of this present study include a small number of patients, very few benign cases, and no control group. These limitations can only be overcome with a larger number of patients with higher number of benign cases as a control group.

\section{Conclusion}

This current prospective study confirmed that EUSFNB of peritoneal lesions appears to be a technically feasible, safe, minimally invasive alternative for tissue diagnosis. The best type of lesion to produce a high yield is a hypoechoic lesion. Moreover, EUS-FNB was found to have a high rate of tissue acquisition adequacy for immunohistochemistry. The technique can avoid more invasive diagnostic laparoscopy in most patients.

\section{Abbreviations \\ EUS: Endoscopic ultrasound; EUS-FNB: Endoscopic ultrasound-guided fine needle biopsy; EUS-FNA: Endoscopic ultrasound-guided fine needle aspira- tion; IHC: Immunohistochemistry stain; CT: Computed tomography; MRI: Magnetic resonance imaging; SD: Standard deviation; PC: Peritoneal carcino- matosis; PPV: Positive predictive value; NPV: Negative predictive value.}

\section{Supplementary Information}

The online version contains supplementary material available at https://doi. org/10.1186/s12876-021-01953-9.

Additional file 1. Video 1: This Video demonstrated a peritoneal lesion being sampled with a 20-gauge needle (EchoTip ProCore 20 gage needle; Cook Medical, Limerick, Ireland). 


\section{Acknowledgements}

Authors thank the research team of the Department of Medicine, Faculty of Medicine, Chulalongkorn University for final editing of the manuscript.

\section{Authors' contributions}

PK designed and created the concept; PK, TO, SY, NS, NK, AS, SS provided the acquisition of clinical and pathological data; PK, TO, SY, NK, AS participated in data analysis and interpretation; PK, TO wrote a draft of the manuscript; All authors read and approved the final manuscript.

\section{Funding}

No Grant support or funding were provided for this study.

\section{Availability of data and materials}

The datasets used and/or analyzed during the current study are available from the corresponding author on reasonable request.

\section{Declarations}

\section{Ethics approval and consent to participate}

The study was approved by the Institutional Review Board of Chulalongkorn University in Bangkok, Thailand with IRB number 422/60 and informed consent was taken from all individual participants.

\section{Consent for publication}

Not applicable.

\section{Competing interests}

The authors declare no conflicts of interest.

\section{Author details}

${ }^{1}$ Excellence Center for Gastrointestinal Endoscopy, King Chulalongkorn Memorial Hospital, Thai Red Cross Society, and Division of Gastroenterology, Department of Medicine, Faculty of Medicine, Chulalongkorn University, Bangkok, Thailand. ${ }^{2}$ Pancreas Research Unit, Department of Medicine, Faculty of Medicine, Chulalongkorn University, Bangkok, Thailand. ${ }^{3}$ Department of Obstetrics and Gynecology, Faculty of Medicine, Chulalongkorn University and King Chulalongkorn Memorial Hospital, Thai Red Cross Society, Bangkok, Thailand. ${ }^{4}$ Department of Pathology, Faculty of Medicine, Chulalongkorn University and King Chulalongkorn Memorial Hospital, Thai Red Cross Society, Bangkok, Thailand.

Received: 30 May 2021 Accepted: 29 September 2021

Published online: 24 October 2021

\section{References}

1. González-Moreno S, González-Bayón L, Ortega-Pérez G, GonzálezHernando C. Imaging of peritoneal carcinomatosis. Cancer J (Sudbury, Mass). 2009;15(3):184-9.

2. Flanagan M, Solon J, Chang KH, Deady S, Moran B, Cahill R, Shields C, Mulsow J. Peritoneal metastases from extra-abdominal cancer-a population-based study. Eur J Surg Oncol J Eur Soc Surg Oncol Br Assoc Surg Oncol. 2018;44(11):1811-7.

3. Fujimura T, Kinami S, Ninomiya I, Kitagawa H, Fushida S, Nishimura G, Kayahara M, Shimizu K, Ohta T, Miwa K. Diagnostic laparoscopy, serum CA125, and peritoneal metastasis in gastric cancer. Endoscopy. 2002;34(7):569-74.

4. Allen VA, Takashima Y, Nayak S, Manahan KJ, Geisler JP. Assessment of false-negative ascites cytology in epithelial ovarian carcinoma: a study of 313 patients. Am J Clin Oncol. 2017;40(2):175-7.

5. DeWitt J, LeBlanc J, McHenry L, McGreevy K, Sherman S. Endoscopic ultrasound-guided fine-needle aspiration of ascites. Clin Gastroenterol Hepatol Off Clin Pract J Am Gastroenterol Assoc. 2007;5(5):609-15.

6. Karoo RO, Lloyd TD, Garcea G, Redway HD, Robertson GS. How valuable is ascitic cytology in the detection and management of malignancy? Postgrad Med J. 2003;79(931):292-4.

7. Kaushik N, Khalid A, Brody D, McGrath K. EUS-guided paracentesis for the diagnosis of malignant ascites. Gastrointest Endosc. 2006;64(6):908-13.
8. McGibbon A, Chen GI, Peltekian KM, van Zanten SV. An evidence-based manual for abdominal paracentesis. Dig Dis Sci. 2007;52(12):3307-15.

9. Blastik M, Plavecz E, Zalatnai A. Pancreatic carcinomas in a 60-year, institute-based autopsy material with special emphasis of metastatic pattern. Pancreas. 2011;40(3):478-80.

10. Pombo F, Rodriguez E, Martin R, Lago M. CT-guided core-needle biopsy in omental pathology. Acta Radiol (Stockholm, Sweden: 1987). 1997;38(6):978-81.

11. Levy MJ, Abu Dayyeh BK, Fujii LL, Clayton AC, Reynolds JP, Lopes TL, Rao AS, Clain JE, Gleeson FC, lyer PG, et al. Detection of peritoneal carcinomatosis by EUS fine-needle aspiration: impact on staging and resectability (with videos). Gastrointest Endosc. 2015;81(5):1215-24.

12. Peter S, Eltoum I, Eloubeidi MA. EUS-guided FNA of peritoneal carcinomatosis in patients with unknown primary malignancy. Gastrointest Endosc. 2009;70(6):1266-70.

13. Somani P, Sharma M, Patil A, Shastri C. Endoscopic ultrasound-guided fine-needle aspiration of peritoneal deposits in patients with ascites of unknown cause (with videos). Endosc Ultrasound. 2017;6(1):69-70.

14. Rimbaş M, Crino SF, Gasbarrini A, Costamagna G, Scarpa A, Larghi A. EUS-guided fine-needle tissue acquisition for solid pancreatic lesions: finally moving from fine-needle aspiration to fine-needle biopsy? Endosc Ultrasound. 2018;7(3):137-40.

15. Iwashita T, Yasuda I, Mukai T, Doi S, Nakashima M, Uemura S, Mabuchi M, Shimizu M, Hatano Y, Hara A, et al. Macroscopic on-site quality evaluation of biopsy specimens to improve the diagnostic accuracy during EUS-guided FNA using a 19-gauge needle for solid lesions: a single-center prospective pilot study (MOSE study). Gastrointest Endosc. 2015;81(1):177-85.

16. Wang J, Wu X, Yin P, Guo Q, Hou W, Li Y, Wang Y, Cheng B. Comparing endoscopic ultrasound (EUS)-guided fine needle aspiration (FNA) versus fine needle biopsy (FNB) in the diagnosis of solid lesions: study protocol for a randomized controlled trial. Trials. 2016;17:198.

17. Vilmann P, Clementsen PF, Colella S, Siemsen M, De Leyn P, Dumonceau JM, Herth FJ, Larghi A, Vazquez-Sequeiros E, Hassan C, et al. Combined endobronchial and esophageal endosonography for the diagnosis and staging of lung cancer: European Society of Gastrointestinal Endoscopy (ESGE) Guideline, in cooperation with the European Respiratory Society (ERS) and the European Society of Thoracic Surgeons (ESTS). Endoscopy. 2015;47(6):545-59.

18. Ishiwatari $\mathrm{H}$, Hayashi $\mathrm{T}$, Kawakami H, Isayama H, Hisai H, Itoi T, Ono M, Kawakubo K, Yamamoto N, Tanaka M, et al. Randomized trial comparing a side-port needle and standard needle for EUS-guided histology of pancreatic lesions. Gastrointest Endosc. 2016;84(4):670-8.

19. Cotton PB, Eisen GM, Aabakken L, Baron TH, Hutter MM, Jacobson BC, Mergener K, Nemcek A Jr, Petersen BT, Petrini JL, et al. A lexicon for endoscopic adverse events: report of an ASGE workshop. Gastrointest Endosc. 2010;71(3):446-54.

20. Facciorusso A, Stasi E, Di Maso M, Serviddio G, Ali Hussein MS, Muscatiello N. Endoscopic ultrasound-guided fine needle aspiration of pancreatic lesions with 22 versus 25 Gauge needles: a meta-analysis. United Eur Gastroenterol J. 2017;5(6):846-53.

21. Facciorusso A, Ramai D, Conti Bellocchi MC, Bernardoni L, Manfrin E, Muscatiello N, Crinò SF. Diagnostic yield of endoscopic ultrasound-guided liver biopsy in comparison to percutaneous liver biopsy: a two-center experience. Cancers. 2021;13(12):3062.

22. Facciorusso A, Sunny SP, Del Prete V, Antonino M, Muscatiello N. Comparison between fine-needle biopsy and fine-needle aspiration for EUSguided sampling of subepithelial lesions: a meta-analysis. Gastrointest Endosc. 2020;91(1):14-22.e12.

23. Facciorusso A, Di Maso M, Serviddio G, Larghi A, Costamagna G, Muscatiello N. Echoendoscopic ethanol ablation of tumor combined with celiac plexus neurolysis in patients with pancreatic adenocarcinoma. J Gastroenterol Hepatol. 2017;32(2):439-45.

24. Rana SS, Bhasin DK, Srinivasan R, Singh K. Endoscopic ultrasound-guided fine needle aspiration of peritoneal nodules in patients with ascites of unknown cause. Endoscopy. 2011;43(11):1010-3.

25. Lee YT, Ng EK, Hung LC, Chung SC, Ching JY, Chan WY, Chu WC, Sung JJ. Accuracy of endoscopic ultrasonography in diagnosing ascites and predicting peritoneal metastases in gastric cancer patients. Gut. 2005;54(11):1541-5. 
26. Runyon BA, Hoefs JC, Morgan TR. Ascitic fluid analysis in malignancyrelated ascites. Hepatology (Baltimore, MD). 1988;8(5):1104-9.

27. Runyon BA, Montano AA, Akriviadis EA, Antillon MR, Irving MA, McHutchison JG. The serum-ascites albumin gradient is superior to the exudatetransudate concept in the differential diagnosis of ascites. Ann Intern Med. 1992;117(3):215-20.

28. Muntean V, Mihailov A, lancu C, Toganel R, Fabian O, Domsa I, Muntean MV. Staging laparoscopy in gastric cancer. Accuracy and impact on therapy. J Gastrointest Liver Dis JGLD. 2009:18(2):189-95.

29. Crinò SF, Di Mitri R, Nguyen NQ, Tarantino I, de Nucci G, Deprez PH, Carrara S, Kitano M, Shami VM, Fernández-Esparrach G, et al. Endoscopic ultrasound-guided fine-needle biopsy with or without rapid on-site evaluation for diagnosis of solid pancreatic lesions: a randomized controlled non-inferiority trial. Gastroenterology. 2021;161:899-909.

30. Crinò SF, Le Grazie M, Manfrin E, Conti Bellocchi MC, Bernardoni L, Granato A, Locatelli F, Parisi A, Di Stefano S, Frulloni L, et al. Randomized trial comparing fork-tip and side-fenestrated needles for EUS-guided fine-needle biopsy of solid pancreatic lesions. Gastrointest Endosc. 2020;92(3):648-658.e642.
31. Facciorusso A, Del Prete V, Antonino M, Buccino VR, Wani S. Diagnostic yield of EUS-guided through-the-needle biopsy in pancreatic cysts: a meta-analysis. Gastrointest Endosc. 2020;92(1):1-8.e3.

32. Larghi A, Manfrin E, Fabbri C, Crinò SF, Correale L, Chiarello G, Barresi L, Van Velthuysen ML, Poley JW, Rahal D, et al. Interobserver agreement among expert pathologists on through-the-needle microforceps biopsy samples for evaluation of pancreatic cystic lesions. Gastrointest Endosc. 2019;90(5):784-792.e784.

33. Binda C, Dabizzi E, Sinagra E, Fornelli A, Saragoni L, Cennamo V, Anderloni A, Fabbri C. Micro-biopsy forceps in the assessment of peritoneal carcinomatosis: a possible new indication? Clin Endosc. 2021;54:613.

\section{Publisher's Note}

Springer Nature remains neutral with regard to jurisdictional claims in published maps and institutional affiliations.
Ready to submit your research? Choose BMC and benefit from:

- fast, convenient online submission

- thorough peer review by experienced researchers in your field

- rapid publication on acceptance

- support for research data, including large and complex data types

- gold Open Access which fosters wider collaboration and increased citations

- maximum visibility for your research: over $100 \mathrm{M}$ website views per year

At BMC, research is always in progress.

Learn more biomedcentral.com/submissions 